\title{
Calculating standard captured $\gamma$ spectra of formation elements
}

\author{
Wu Wensheng*, Xiao Lizhi, Zhang Lijuan and Niu Wei \\ State Key Laboratory of Petroleum Resource and Prospecting, China University of Petroleum, Beijing 102249, China \\ (C) China University of Petroleum (Beijing) and Springer-Verlag Berlin Heidelberg 2012
}

\begin{abstract}
Multi-element gamma-ray spectrum logging requires standard spectra of individual elements for its interpretation. Since the standard element spectra are usually derived using Monte Carlo simulation of either a pure element or its oxide, we synthesized the standard neutron captured $\gamma$ spectra (as would be observed using a $\mathrm{NaI}(\mathrm{Tl})$ detector) of elements $\mathrm{H}, \mathrm{Si}, \mathrm{Ca}$ and $\mathrm{Fe}$ from each element and its oxide. To compare the standard spectra from the elements and oxides, we operated three simulations of sandstone, limestone and mixed formation of sandstone and limestone each with ten different porosities, and used the two kinds of standard spectra to analyze the mixed spectra modeled from sandstone and limestone formations. The results show that the standard element spectra from oxides have more prominent energy peaks than the standard spectra from pure elements. The calculated formation element contents are close to the theoretical values when the standard element spectra from oxides are used to analyze the formation mixed spectra. Therefore, the formation element standard spectra should be calculated from oxide models in the analysis of neutron captured $\gamma$ spectra by logging tools.
\end{abstract}

Key words: Standard spectrum, spectrum analysis, neutron captured $\gamma$ spectrum, formation element

\section{Introduction}

Multi-element spectrum logging can effectively identify lithology and mineralogy in formations, and plays an important role in exploring complex reservoirs (Hertzog et al, 1989; Chapman et al, 1987; Grau and Schweitzer, 1989; Grau et al, 1990). Accurate and effective full spectrum analysis is one of the key technologies in full spectrum captured $\gamma$ logging, and the spectrum analysis method requires simulated standard captured $\gamma$ spectra of major formation elements.

There are two ways to acquire standard element spectra, one is instrument measurement in a standard calibration model, and the other is numerical simulation (Nguyen et al, 1996; Al-Ghorabie, 2006; Shi et al, 2002; Xiang and Guo, 2006). As numerical simulation can not completely take the real tool and well conditions into account, it is better to obtain the standard spectra with a spectrum tool in a model well. Owing to a variety of reasons, almost all publications do not describe elements or compounds used to synthesize standard element spectra in the model well. Generally, either a pure element or its oxide can be used to obtain standard element spectra by Monte Carlo modeling.

With the Monte Carlo numerical simulation program MCNP(5C) (Pei and Zhang, 1980), we studied the differences of spectrum analysis using standard spectra calculated either from pure elements or their oxides.

*Corresponding author. email: wwsheng@yahoo.com.cn Received January 5, 2012

\section{Numerical simulation model}

The calculation model is a cylinder with a height of $1 \mathrm{~m}$ and a radius of $70 \mathrm{~cm}$, as shown in Fig. 1. The diameter of the logging tool with a pulsed neutron source is $45 \mathrm{~mm}$. The source emits $14 \mathrm{MeV}$ neutrons into the formation with a pulse width of $40 \mu$ s and a period of $100 \mu$ s. There is a NaI(Tl) detector $40 \mathrm{~cm}$ away from the source and the detector is 10 $\mathrm{cm}$ in length and $4 \mathrm{~cm}$ in diameter. The tool is pressed closely up against one side of the borehole. The space between the source and the detector is filled with shielding material. The detector responses are considered in the calculation.

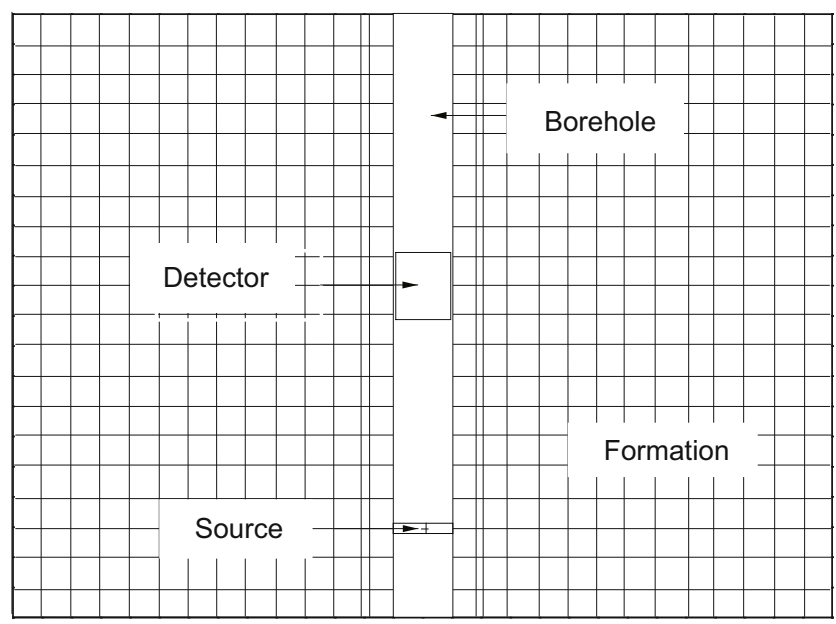

Fig. 1 The calculation model 
A two-step simulation is used in the calculation. First is the simulation for neutrons. When photons are generated, the position where photons are generated is treated as the departure position. The energy and motion direction of the photons are determined by random sampling of the known energy distribution and motion direction distribution, thus the second step simulation begins (Hendriks et al, 2002). The advantages of this two-step simulation are significant whole energy, single escape and double escape peaks, short computation time, and low calculation error generally less than $0.1 \%$.

\section{The simulation for standard element spectra in formation}

\subsection{The simulation for the formation filled with pure elements}

With the above calculation model and calculation techniques, the next most critical step is filling the element in the model for standard spectrum simulation. Since the standard element spectrum is the detector response of the logging tool to a transient nuclear reaction of atomic nuclei in the borehole, pure substances are first considered to be filled in the model to simulate element standard spectra. In computation, the pure element in the model is respectively $\mathrm{H}$, $\mathrm{Si}, \mathrm{Ca}$ and $\mathrm{Fe}$, and the calculated captured $\gamma$ spectra are shown in Fig. 2. The inelastic scattered gamma counts are deducted from the captured spectra counts in Fig. 2. Because the spectra are obtained between two neutron pulses, the spectra in the figure are almost free from the influence of activation gamma rays.

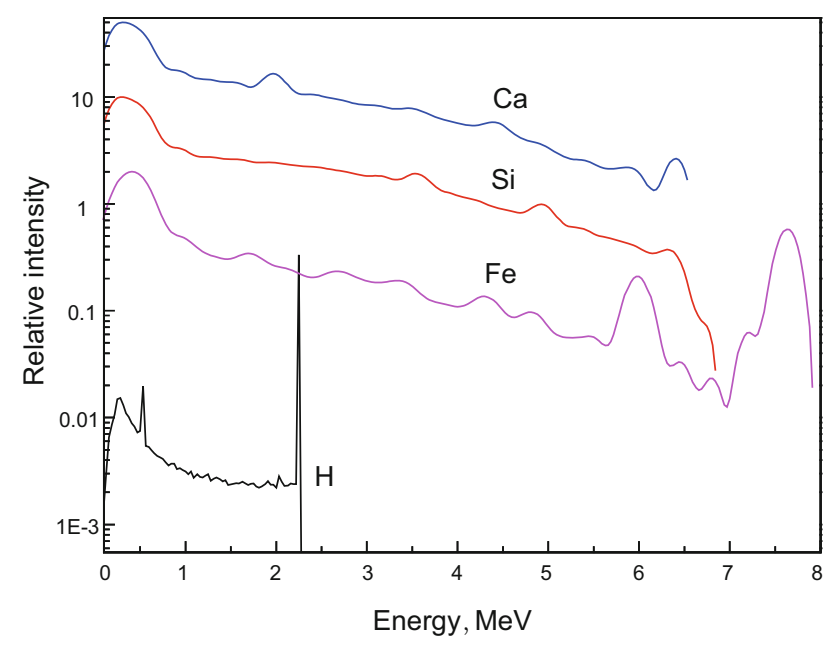

Fig. 2 Captured $\gamma$ spectra of $\mathrm{H}, \mathrm{Si}, \mathrm{Ca}$ and $\mathrm{Fe}$

Fig. 2 shows that there is a characteristic peak of $\mathrm{H}$ at $2.23 \mathrm{MeV}$; two whole energy peaks of Si appear at $3.54 \mathrm{MeV}$ and $4.93 \mathrm{MeV}$; Ca peaks are at $1.94 \mathrm{MeV}$ and $6.42 \mathrm{MeV}$; $\mathrm{Fe}$ peaks are at $7.46 \mathrm{MeV}$ and $5.92 \mathrm{MeV}$ (Huang, 1985). In the figure, the first peak on the left is the backscattered peak. Some of the $\gamma$-rays arriving at the detector pass completely through the detector crystal without being detected. They then interact with the atoms of the materials behind the detector (Compton effect) and the resultant backscattered $\gamma$-rays arrive back at the detector, are detected and form the backscattered low-energy peak. As well, backscattered photons produced in shielding materials also contribute to the backscattered peak. The energy of backscattered photons is always around $200 \mathrm{keV}$, so it is easy to identify the backscattered peak in the spectrum.

\subsection{The simulation for the formation filled with element oxide}

Considering the element in the strata is not a pure element, but an oxide form, for example, element $\mathrm{H}$ occurs as $\mathrm{H}_{2} \mathrm{O}$, and $\mathrm{Si}$ occurs as $\mathrm{SiO}_{2}$, the model is filled with the oxides $\mathrm{H}_{2} \mathrm{O}$, $\mathrm{SiO}_{2}, \mathrm{CaCO}_{3}$, and $\mathrm{FeO}$, and the calculated captured $\gamma$ spectra are shown in Fig. 3.

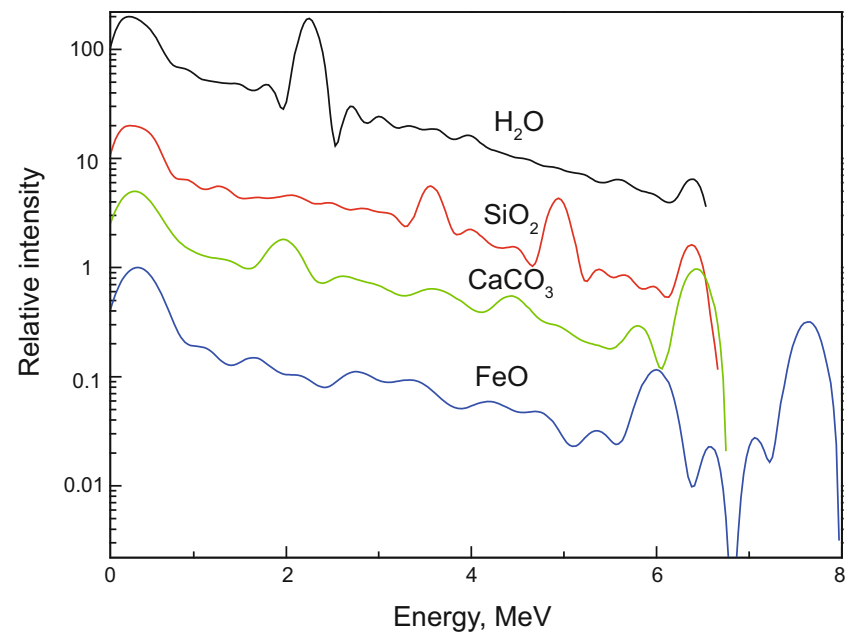

Fig. 3 Captured $\gamma$ spectra of the oxides of $\mathrm{H}, \mathrm{Si}, \mathrm{Ca}$ and $\mathrm{Fe}$

To compare the differences of spectra in Fig. 2 and Fig. 3 , the spectra of $\mathrm{Si}$ and $\mathrm{SiO}_{2}$ are taken as an example shown in Fig. 4. Fig. 4 shows that compared with the spectrum of pure element $\mathrm{Si}$, the characteristic peak positions of the $\mathrm{SiO}_{2}$ spectrum do not change but its peaks are more prominent, especially the characteristic peak at $4.93 \mathrm{MeV}$. The characteristic peaks in the spectra of other oxides are also more prominent than those in corresponding pure element spectra. Some small peaks in $\mathrm{SiO}_{2}$ spectrum are due to the presence of element $\mathrm{O}$. The standard captured $\gamma$ spectrum of Si can be obtained by doing mathematical algorithm processing to remove the oxygen peaks from the spectrum of $\mathrm{SiO}_{2}$.

\section{Comparison of energy spectrum analysis results}

\subsection{Sandstone with different porosities}

In order to compare the spectrum analysis results based on the above two types of element standard spectra, it is assumed that there is a sandstone reservoir with different porosities from $1 \%$ to $45 \%$ corresponding to ten points, and the pore is 


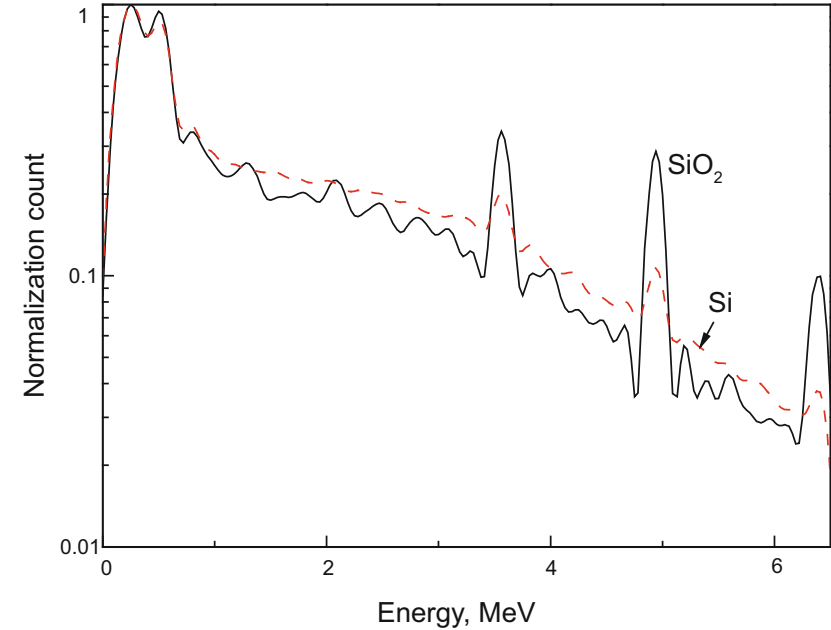

Fig. 4 Comparison of $\mathrm{Si}$ and $\mathrm{SiO}_{2}$ spectra

filled with water, as shown in Table 1 . In Table $1, C_{\mathrm{H}}$ and $C_{\mathrm{Si}}$ represent the weight percentage of $\mathrm{H}$ and $\mathrm{Si}$ respectively, and $\rho_{\mathrm{b}}$ is formation density.

Table 1 Sandstone formation model with different porosities

\begin{tabular}{ccccc}
\hline Point & $\begin{array}{c}C_{\mathrm{H}} \\
\mathrm{wt} \%\end{array}$ & $\begin{array}{c}C_{\mathrm{Si}} \\
\mathrm{wt} \%\end{array}$ & $\begin{array}{c}\text { Porosity } \\
\%\end{array}$ & $\begin{array}{c}\rho_{\mathrm{b}} \\
\mathrm{g} / \mathrm{cm}^{3}\end{array}$ \\
\hline 1 & 0.01 & 36.70 & 1 & 2.634 \\
2 & 0.06 & 36.12 & 5 & 2.568 \\
3 & 0.12 & 35.36 & 10 & 2.485 \\
4 & 0.19 & 34.54 & 15 & 2.403 \\
5 & 0.26 & 33.67 & 20 & 2.320 \\
6 & 0.34 & 32.73 & 25 & 2.238 \\
7 & 0.42 & 31.71 & 30 & 2.155 \\
8 & 0.51 & 30.62 & 35 & 2.073 \\
9 & 0.61 & 29.44 & 40 & 1.990 \\
10 & 0.71 & 28.15 & 45 & 1.908 \\
\hline
\end{tabular}

The program MCNP (5C) is used to simulate the captured $\gamma$ spectra for the ten points in Table 1. Before spectrum analysis, the element standard spectra are isolated from the oxide spectra by mathematical processing, and these element standard spectra are called oxide standard spectra. The standard spectra assuming pure elements are called pure element standard spectra. To conveniently perform mathematical processing, the standard spectra and formation mixed spectra are expressed as 256-dimensional vector $\mathbf{P}$ and the components are normalized according to Eq. (1)

$$
\sum_{i=1}^{256} \boldsymbol{P}_{i}=1
$$

With oxide standard spectra and pure element standard spectra, a full spectrum least-square method is used to analyze the mixed spectra for the ten points in Table 1 and obtain the relative yields of $\mathrm{H}$ and $\mathrm{Si}$. In order to calculate the weight percentage of each element with the oxide model, each point in Table 1 is calibrated to get the normalization factor $F$ and sensitivity factor $S$, and then the weight percentages of elements at each point are calculated by Eq. (2) (Pang et al,
2005; Pang and Li, 2006):

$$
W t_{j}=F \cdot y_{j} / S_{j}
$$

Fig. 5 and Fig. 6 show the weight percentages of $\mathrm{H}$ and Si acquired respectively with the oxide standard spectrum and pure element standard spectrum. The $x$-axis indicates the theoretical composition of the element in the formation, the $y$-axis represents the calculated element content, and the diagonal dashed line in the figure denotes that the calculated value is equal to the theoretical value.

Fig. 5 shows that the $\mathrm{H}$ content obtained from a pure element standard spectrum is higher than the corresponding theoretical value. The content of $\mathrm{H}$ based on oxide standard spectrum is lower than the corresponding theoretical value for low porosities, and higher than the corresponding theoretical value for high porosities. On the whole, the analysis results using the oxide standard spectrum are closer to the diagonal dashed line than those of the pure element standard spectrum, which demonstrates that spectrum analysis with the standard spectrum calculated from the oxide can provide a more accurate estimate of the hydrogen content.

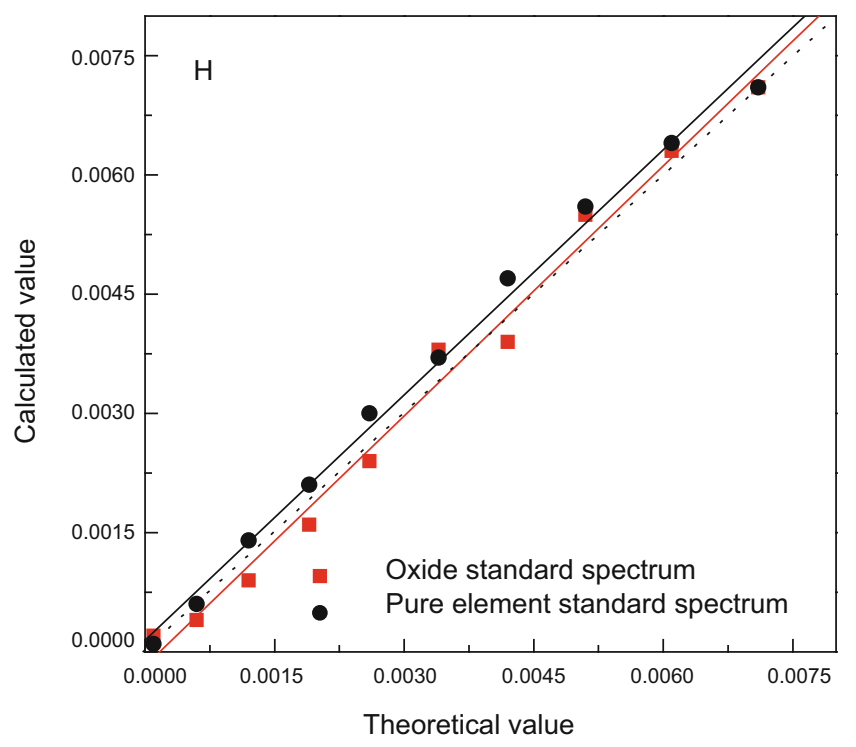

Fig. 5 Weight percentage of $\mathrm{H}$ based on sandstone model

Fig. 6 shows that the content of Si calculated from the oxide standard spectrum is extremely close to the corresponding theoretical value, and the content derived from the pure element standard spectrum is lower than the corresponding theoretical value. The lower the formation porosity, the higher the deviation degree.

Therefore, according to the spectrum analysis results in sandstone formation, the computation accuracy of element content with oxide standard spectrum is better than that with pure element standard spectrum.

\subsection{Limestone with different porosities}

To compare the spectrum analysis results for oxide standard spectrum and pure element standard spectrum, it is assumed that there is a limestone reservoir with the porosity 


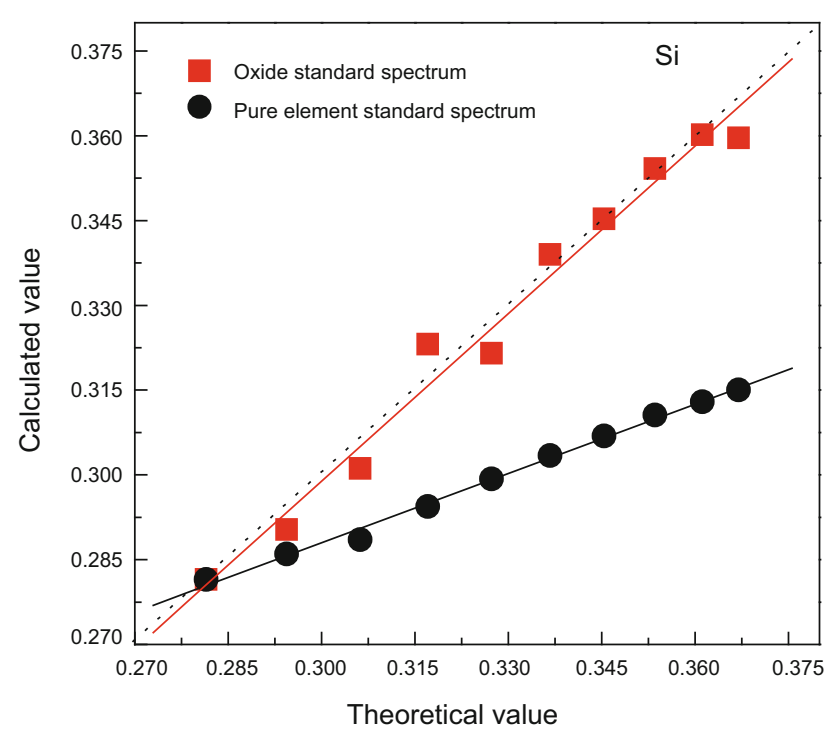

Fig. 6 Weight percentage of Si based on sandstone model

from $1 \%$ to $45 \%$ corresponding to ten points, and the pores are also completely filled with water, as shown in Table 2 . In Table $2, C_{\mathrm{H}}$ and $C_{\mathrm{Ca}}$ stand for the weight percentages of $\mathrm{H}$ and $\mathrm{Ca}$ respectively.

Table 2 Limestone formation model with different porosities

\begin{tabular}{ccccc}
\hline Point & $\begin{array}{c}C_{\mathrm{H}} \\
\mathrm{wt} \%\end{array}$ & $\begin{array}{c}C_{\mathrm{Ca}} \\
\mathrm{wt} \%\end{array}$ & $\begin{array}{c}\text { Porosity } \\
\%\end{array}$ & $\begin{array}{c}\rho_{\mathrm{b}} \\
\mathrm{g} / \mathrm{cm}^{3}\end{array}$ \\
\hline 1 & 0.04 & 31.70 & 1 & 2.693 \\
2 & 0.21 & 31.21 & 5 & 2.625 \\
3 & 0.44 & 30.57 & 10 & 2.539 \\
4 & 0.68 & 29.87 & 15 & 2.454 \\
5 & 0.94 & 29.13 & 20 & 2.368 \\
6 & 1.22 & 28.33 & 25 & 2.283 \\
7 & 1.52 & 27.47 & 30 & 2.197 \\
8 & 1.84 & 26.54 & 35 & 2.112 \\
9 & 2.19 & 25.54 & 40 & 2.026 \\
10 & 2.58 & 24.44 & 45 & 1.941 \\
\hline
\end{tabular}

The analysis results with an oxide standard spectrum and pure element standard spectrum for the mixed spectra at ten points in Table 2 are shown in Fig. 7 and Fig. 8.

Fig. 7 shows that the intersections of calculated content of $\mathrm{H}$ using an oxide standard spectrum and the theoretical content are almost all located on the diagonal line, which means the calculated value is almost the same as the theoretical one. The content of $\mathrm{H}$ obtained from the pure element standard spectrum is much lower than the theoretical one, and the higher the formation porosity, the larger the difference between the calculated value and the theoretical value. These show that the accuracy of $\mathrm{H}$ content calculated from the oxide standard spectrum is relatively high.

In Fig. 8, although the content of $\mathrm{Ca}$ obtained from an oxide standard spectrum is slightly higher than the theoretical

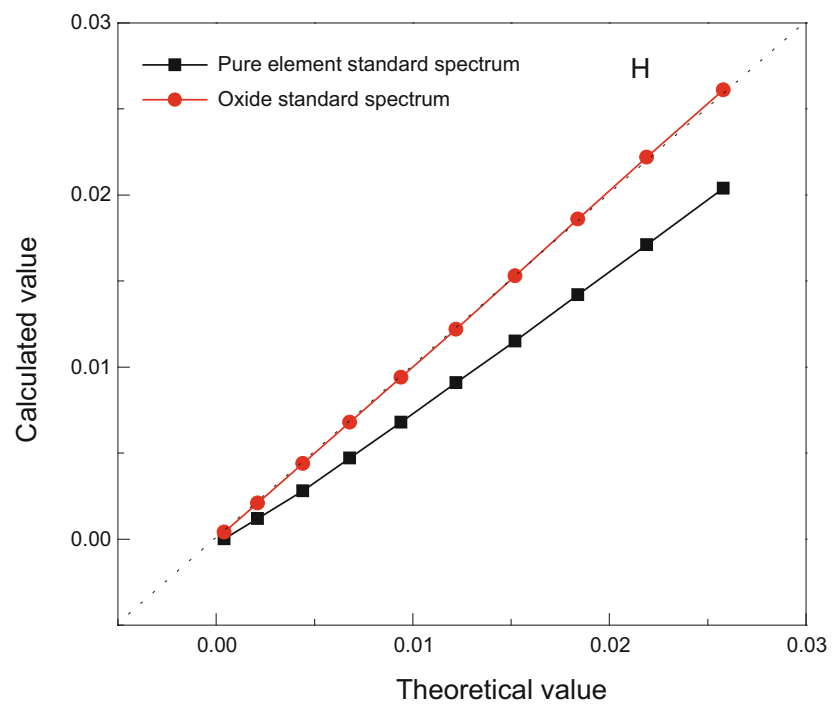

Fig. 7 Weight percentage of $\mathrm{H}$ based on limestone model

value, it is still close to it. The content of $\mathrm{Ca}$ from a pure element standard spectrum is significantly higher than the corresponding theoretical one.

Therefore, from the spectrum analysis results in the limestone formation, the accuracy of the element content calculated from the oxide standard spectrum is better than that from the pure element standard spectrum.

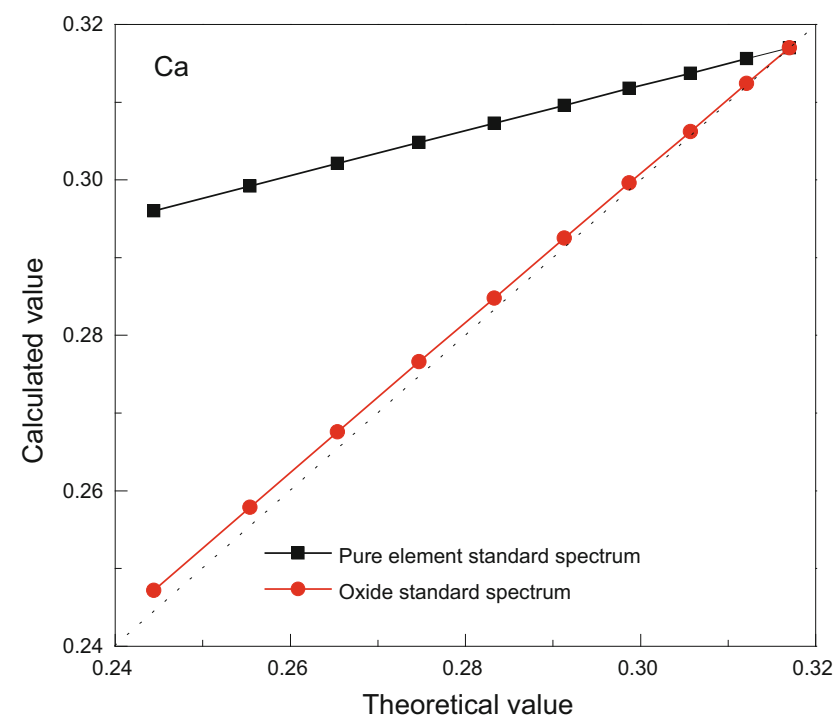

Fig. 8 Weight percentage content of Ca based on limestone model

\subsection{Mixed formation of sandstone and limestone with different porosities}

To further compare the spectrum analysis results in complex formations with the above two types of standard spectra, we suppose that there is a reservoir with the lithology of limestone and sandstone with porosity from $2 \%$ to $10 \%$ corresponding to nine points, and the formation pores are completely filled with water, as shown in Table $3 . C_{\mathrm{H}}, C_{\mathrm{Si}}$ and $C_{\mathrm{Ca}}$ in Table 3 represent the weight percentages of $\mathrm{H}, \mathrm{Si}$ and $\mathrm{Ca}$ respectively. 
Table 3 Mixed formation model of sandstone and limestone

\begin{tabular}{cccccc}
\hline Point & $\begin{array}{c}C_{\mathrm{Si}} \\
\mathrm{wt} \%\end{array}$ & $\begin{array}{c}C_{\mathrm{Ca}} \\
\mathrm{wt} \%\end{array}$ & $\begin{array}{c}C_{\mathrm{H}} \\
\mathrm{wt} \%\end{array}$ & $\begin{array}{c}\text { Porosity } \\
\%\end{array}$ & $\begin{array}{c}\rho_{\text {matrix }} \\
\mathrm{g} / \mathrm{cm}^{3}\end{array}$ \\
\hline 1 & 0.046 & 0.320 & 0.004 & 10 & 2.433 \\
2 & 0.092 & 0.285 & 0.004 & 9 & 2.454 \\
3 & 0.138 & 0.250 & 0.003 & 8 & 2.475 \\
4 & 0.184 & 0.214 & 0.003 & 7 & 2.496 \\
5 & 0.231 & 0.178 & 0.002 & 6 & 2.517 \\
6 & 0.278 & 0.142 & 0.002 & 5 & 2.539 \\
7 & 0.326 & 0.106 & 0.002 & 4 & 2.560 \\
8 & 0.374 & 0.070 & 0.001 & 3 & 2.581 \\
9 & 0.422 & 0.033 & 0.001 & 2 & 2.602 \\
\hline
\end{tabular}

Fig. 9 and Fig. 10 show the analysis results with oxide standard spectrum and pure element standard spectrum for the mixed spectra at nine points in Table 3 .

As shown in Fig. 9, when the content of $\mathrm{Si}$ is higher than 0.2 , the calculated value using pure element standard spectrum is higher than the theoretical one, and the higher the content of $\mathrm{Si}$, the higher the deviation degree from the theoretical value. When the content of $\mathrm{Si}$ is less than 0.2 , the content of $\mathrm{Si}$ obtained from a pure element standard spectrum is lower than the theoretical value, and even negative. However, the intersections of the calculated content of $\mathrm{H}$ using oxide standard spectra and the theoretical content are almost all distributed along the diagonal line, which means the calculated value is extremely close to the model composition. Fig. 10 shows information similar to that of Fig. 9.

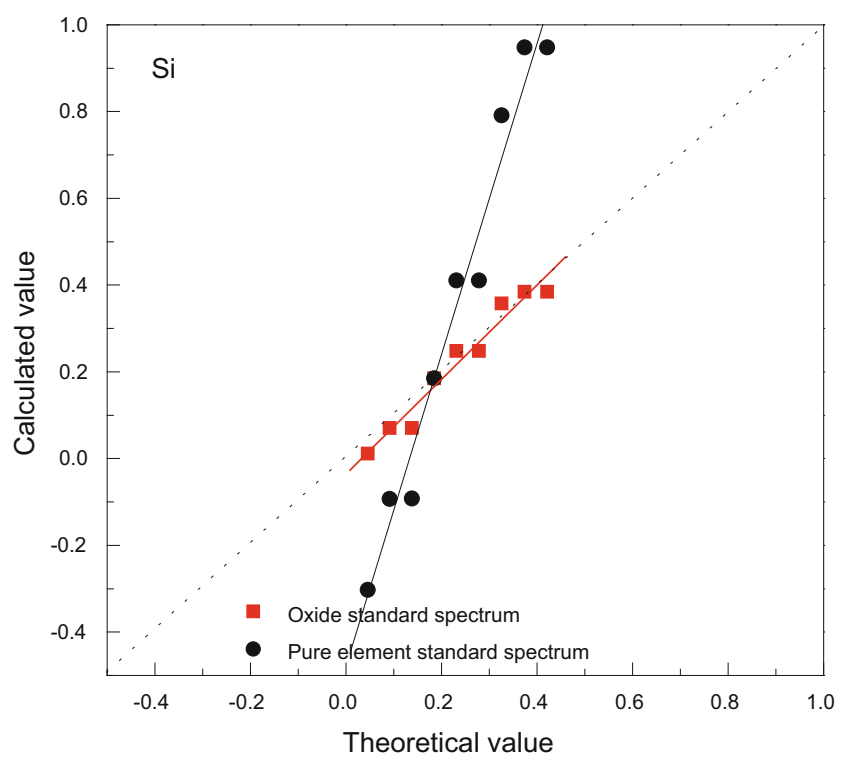

Fig. 9 Weight percentage of Si based on mixed formation

The same can be seen from the analysis results of mixed formation spectra that the computation accuracy of element content using the oxide standard spectrum is better than that with a pure element standard spectrum.

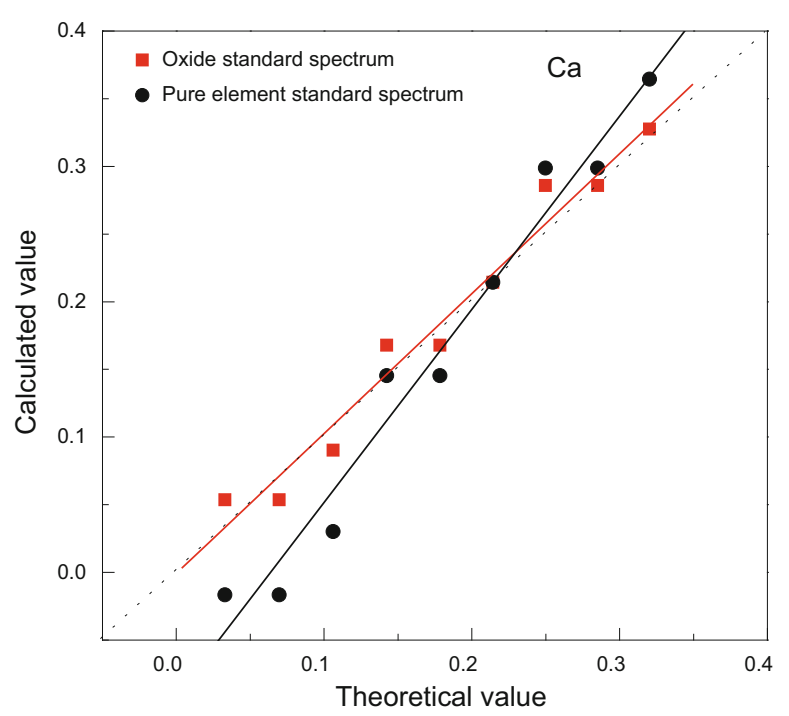

Fig. 10 Weight percentage of Ca based on mixed formation

\section{Conclusions}

The standard spectra of formation elements can be calculated using either pure elements or their oxides. Compared with the standard spectra using a pure element, the standard spectra using the oxide have more prominent characteristic energy peaks. It can be seen from the analysis results of different formation models that the element content calculated with oxide standard spectrum is close to the theoretical value. Therefore, element standard spectra should be calculated from the oxides.

\section{Acknowledgements}

This paper is financially supported by National Natural Science Foundation of China (Grant No. 41074101) and Science Foundation of China University of Petroleum (Beijing) (No. KYJJ2012-05-12).

\section{References}

Al- Ghorabie F H H. Development of a computer code using the EGS4 Monte Carlo simulation system to evaluate the response of a $\mathrm{NaI}(\mathrm{Tl})$ detector to photons with energies below $300 \mathrm{keV}$. Applied Radiation and Isotopes. 2006. 64(1): 85-92

Chapman S, Colson J L, Everett B, et al. The emergence of geochemical well logging. The Technical Review. 1987. 35(2): 27-35

Grau J A and Schweitzer J S. Elemental concentrations from thermal neutron capture gamma-ray spectra in geological formations. Nuclear Geophysics. 1989. 3(1): 1-9

Grau J A, Schweitzer J S and Hertzog R C. Statistical uncertainties of elemental concentrations extracted from neutron induced gamma-ray measurements. IEEE Transactions on Nuclear Science. 1990. 37(6): 2175-2178

Hendriks P H G M, Maucec M and De Meijer R J. MCNP modeling of scintillation-detector $\gamma$-ray spectra from natural radionuclides. Applied Radiation and Isotopes. 2002. 57(3): 449-457

Hertzog R, Colson L, Seeman O, et al. Geochemical logging with spectrometry tools. SPE Formation Evaluation. 1989. 4(2): 153-162 (SPE16792)

Huang L J. The Principle of Radioactive Logging. Beijing: Petroleum 
Industry Press. 1985. appendix (in Chinese)

Nguyen H V, Campbell J M, Couchell G P, et al. Programs in C for parameterizing measured $5^{\prime \prime} \times 5^{\prime \prime} \mathrm{NaI}$ gamma response functions and unfolding of continuous gamma spectra. Computer Physics Communications. 1996. 93(2-3): 303-321

Pang J F and Li M. Analytical methods with neutron-gamma ray spectrometry from formation elements. Journal of Isotopes. 2006. 19(2): 70-74 (in Chinese)

Pang J F, Li M and Yan Z G. New analyzing method \& software and applications of inelastic spectra from $\mathrm{C} / \mathrm{O}$ spectrometry logs. Nuclear
Physics Review. 2005. 22(1): 67-71 (in Chinese)

Pei L C and Zhang X Z. The Monte Carlo Method and Its Application in Particle Transport. Beijing: Science Press. 1980. 5-50 (in Chinese)

Shi H X, Chen B X, Li T Z, et al. Precise Monte Carlo simulation of gamma-ray response functions for a $\mathrm{NaI}(\mathrm{Tl})$ detector. Applied Radiation and Isotopes. 2002. 57(4): 517-524

Xiang D and Guo L Y. Monte Carlo simulation of gamma spectra. Journal of Mathematical Medicine. 2006. 19(3): 229-231 (in Chinese)

(Edited by Hao Jie) 